\title{
Study of Sequencing Batch Reactor operation with an inflow of wastewater with low concentration of organic contaminations
}

\author{
Elena Gogina ${ }^{1, *}$ \\ ${ }^{1}$ NRU Moscow state university of civil engineering (NRU MGSU), 129337, Jaroslavskoje sch. 26
}

\begin{abstract}
The paper is devoted to the study of small wastewatertreatment plants, in particular the study of wastewater treatment processes with the wastewater with low concentration of organic contaminations entering treatment plants. The primary goal of the study is to select the reactor operation mode to ensure the deep biological treatment quality of treated wastewater. The three-step experiment has been conducted in a licensed laboratory using high quality equipment. The results of the experiment and the analysis of the data obtained are presented.
\end{abstract}

\section{Introduction}

The environmental pollution and the contamination of water bodies in particular is the major global problem at the moment. Wastewater treatment facilities are one of the main sources of pollution, due to the fact that new wastewater treatment plants are scarcely being built, and the technical re-equipment and reconstruction of industrial and municipal wastewater treatment facilities is one of the most difficult engineering tasks [1-4].

The amount of wastewater delivered to aeration and activated sludge plants in the Russian Federation in many cases exceeds their rated capacity, resulting in deteriorating treatment quality and sanitary conditions of water bodies. In some cases, which have become more frequent recently, the pace of housing construction is constrained by the lack of reserve and unwillingness to reconstruct drainage systems and wastewater treatment facilities. The situation is further aggravated by the lack of available space for the expansion of the treatment plants complex. Therefore, it is necessary to reduce the cost of reconstruction methods as much as possible and place the reconstructed facilities in the previously allocated areas. These issues require significant innovations. Such innovations include a Sequencing Batch Reactor (SBR), which allows to combine two facilities: an aerotank and a secondary sedimentation tank. If concentration of suspended solids and organic matter in wastewater is low, there will be no need for a primary sedimentation tank. This will allow doubling the capacity of the wastewater treatment plant without using additional territory [5-8].

The use of SBR technology allows to easily regulate and, if necessary, quickly change the time of stay of treated water in the bioreactor, the activated sludge concentration, the

* Corresponding author: goginaes@mgsu.ru 
sludge load, its age, the concentration of dissolved oxygen, sedimentation time, loading and unloading. All technological operations in the bioreactor can be performed either according to a given time program or according to the oxygen concentration sensor, i.e. oxygen consumption.

The aim of this paper is to study the Sequencing Batch Reactor operation mode and to select the most suitable technological mode of reactor operation with an inflow of wastewater with low concentration of organic contaminations.

The experiment consisted of studying the Sequencing Batch Reactor (SBR) operation modes, using a laboratory model, and conducting a series of sanitary and chemical analyses.

The sequencing batch reactor (SBR) technology of wastewater treatment is quite promising and widespread in Western Europe. The system is based on the Sequencing Batch Reactor. Unlike traditional methods when water flows through several tanks or chambers, in the SBR system all cyclic stages of purification take place in one tankbioreactor. The SBR system operates on the principle of activated sludge accumulation. In the bioreactor, all contamination from the drain is absorbed by floating activated sludge microorganisms and turns into biomass.

The principle of the SBR operation is based on a cyclic change of wastewater treatment processes occurring in one reactor. Each cycle consists of certain phases, the duration and sequence of which is set according to the required biological treatment effect.

The main advantage of SBR is that all biological treatment processes (oxidation of the organics and nutrients, as well as sedimentation) take place in the same reactor, which makes it possible to regulate the duration and sequence of the treatment phases based on the concentration of wastewater, entering treatment facilities, with the use of automated control systems [9-14].

This technology allows receiving drainage water with high coefficient of flow irregularity and scarcely depend on the quality of the incoming water. The use of SBR technology allows to easily regulate and, if necessary, quickly change the time of stay of treated water in the bioreactor, the activated sludge concentration, the sludge load, its age, the concentration of dissolved oxygen, sedimentation time, loading and unloading. All technological operations in the bioreactor can be performed either according to a given time program or according to the oxygen concentration sensor, i.e. oxygen consumption.

The second peculiar feature of the SBR technology is the retention of the settled activated sludge in the bioreactor after the end of the wastewater treatment period. The settled sludge volume amounts to $35-45 \%$ of the total volume of the bioreactor, its ash-free biomass matter concentration is in the range from 12 to $15 \mathrm{~kg} / \mathrm{m}^{3}$. By selecting or retaining excess sludge in the bioreactor, the concentration of activated sludge is being corrected with each new portion of the treated drainage water. Thus, the working concentration of activated sludge, its age and sludge load are regulated within the necessary limits corresponding to changes in composition or concentration of contaminations in the wastewater.

The third feature of the SBR technology is an autonomous system of sludge mix aeration in the bioreactor. Aeration is performed by mechanical turboaerators on a floating platform or by bottom hyperbolic stirrers with air supply from an individual compressor. Due to the use of the variable-frequency drive, the speed of turbines or stirrers can vary in a wide range and at very low speeds $(2,050 \mathrm{rpm})[15,16]$.

The fourth feature of the SBR technology is a cyclic schedule of air supply to the bioreactor with alternating periods of intensive sludge mix aeration (organics bio-oxidation and nitrification) and periods of slow mixing without oxygen supply, denitrification, biological phosphorus removal). Depending on the concentration of contaminations in the 
wastewater, the sludge mix oxidation schedule can be changed rapidly, e.g. increasing the aeration time by reducing the mixing time or vice versa.

Another distinguishing feature of the SBR technology is that the same type of bioreactor can be used for wastewater treatment with different operating cycles: $8,9,12,15,16,18$, 21 or 24 hours depending on the degree of wastewater contamination and the contaminations oxidation degree [17].

Having studied the current Sequencing Batch Reactor, one can conclude that the SBR technology has not been fully explored. Nowadays, the most suitable mode of operation of the Sequencing Batch Reactor with an inflow of wastewater with low concentration of organic contaminations has not yet been developed.

\section{Materials and Methods}

The study consisted of three parts. The tests were carried out at the laboratory bench of the Scientific Educational Center "Water Supply and Water-Removal" of the Water Supply and Water-Removal Department of the Moscow State (National Research) University of Civil Engineering. A 30 litres plexiglass column was used in the biological reactor operation. In order to find the most suitable modes of operation, outlets were mounted in this column at different heights. A compressor, supplying air through aerators, was used to create the aeration mode, and a stirrer was used to create the mixing mode. In order to optimize the column performance as a Sequencing Batch Reactor, time relays were used, which switched on and off the aerator, stirrers in accordance with the specified time, and created conditions for the activated sludge sedimentation. The laboratory-scale plant operation diagram is shown in Fig. 1. Then there is a photo of the plant in Fig. 2.

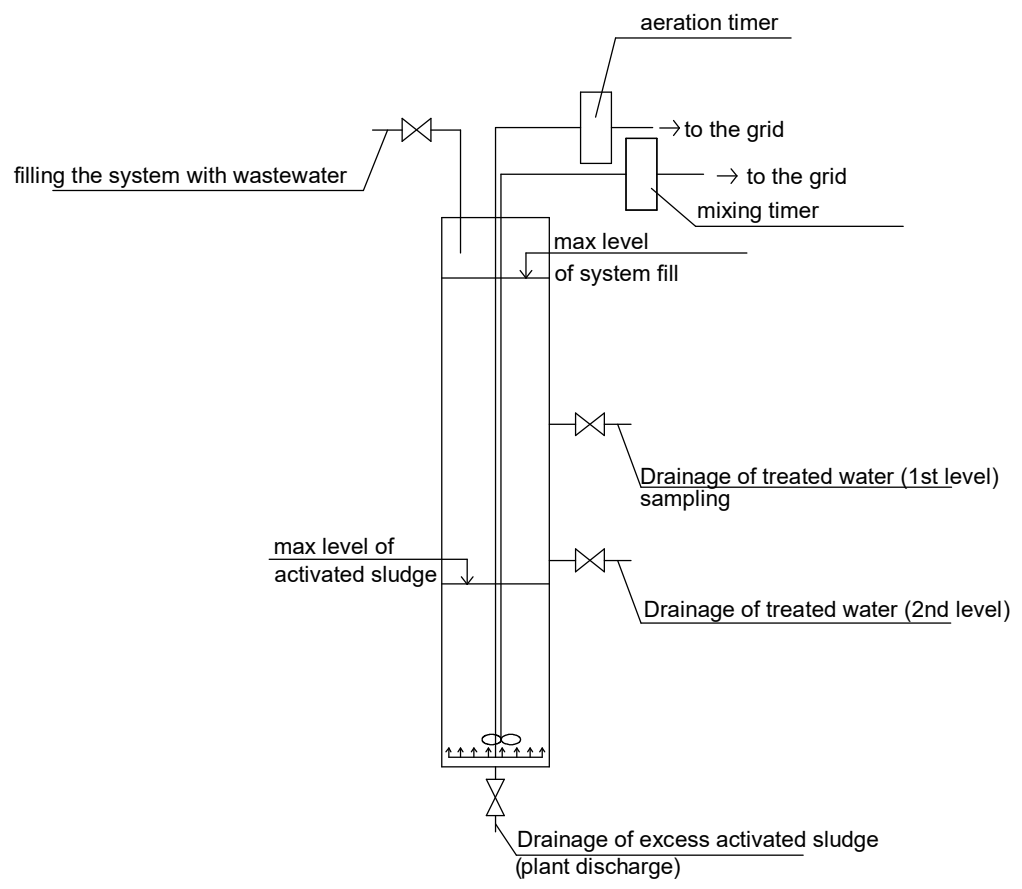

Fig. 1. The diagram of the laboratory model to study the Sequencing Batch Reactor operation. 


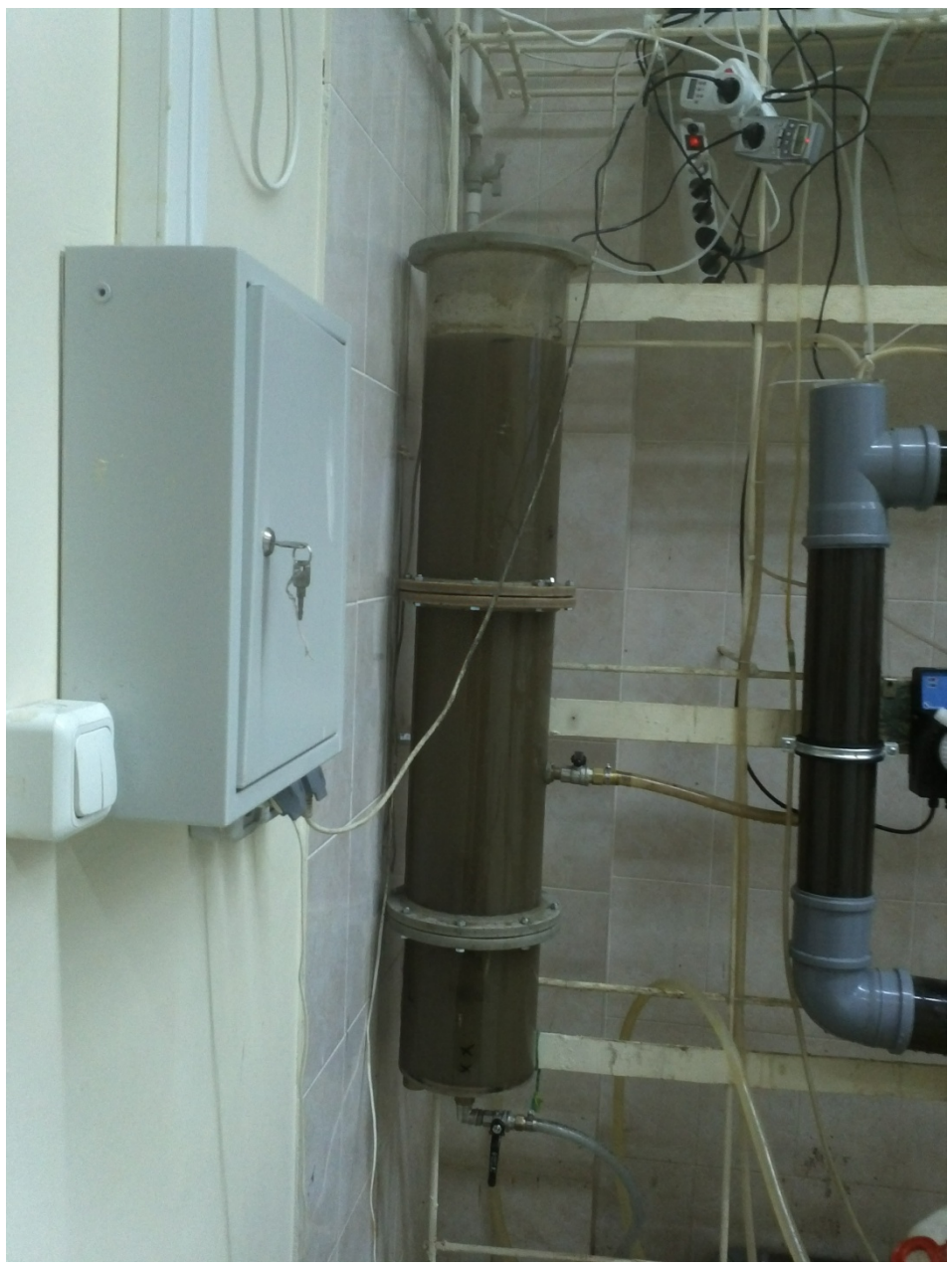

Fig. 2. A photo of the Sequencing Batch Reactor plant.

The work was carried out with the use of artificially composed peptone-based waste liquid. The contaminations concentrations were closest to those existing in the actual waste liquid. In the course of the experiment, the concentrations of certain substances and the hydraulic load on the plant changed depending on the task assigned. The main parameters of the waste liquid entering the treatment plant are given below:

Average concentration of contaminations in wastewater

$-\mathrm{BOD}_{5}-110 \mathrm{mg} / \mathrm{L}$

- Ammonium nitrogen $\left(\mathrm{NH}_{4}^{-}\right)-28 \mathrm{mg} / \mathrm{L}$

- Suspended substances $-90 \mathrm{mg} / \mathrm{L}$.

\section{Conducting an experiment}

The experiment was carried out on a pilot plant in three stages, with the aim of finding the optimal mode of operation of the Sequencing Batch Reactor with wastewater with low concentration of organic contaminations. Each stage corresponded to a certain technological mode. The modes of operation differed by the operating time of the plant, as well as the duration of the phases of aeration and mixing. 
First stage. During the first part of the experiment, the reactor operation mode was set at 8 hours for 15 days. The artificially composed waste liquid was as close to the real waste liquid as possible in its parameters. Wastewater consumption of the plant was $0.2 \mathrm{~m}^{3} /$ day.

The reactor operation mode included following cycles:

1. Filling the system with wastewater;

2. 2 hours - sludge mix aeration, 1 hour - mixing, then 2 hours - aeration and 1 hour mixing;

3. Sedimentation of the purified water for 2 hours;

4. Drainage of the purified water;

5. Drainage of the excess activated sludge.

During the experiment, after each cycle, a series of sanitary-chemical analyses were conducted, which showed poor analyses results. The reactor is ineffective in this mode. BOD decreased by $90.9 \%$ at this stage, which indicates that there is not enough time for oxidation of organic contaminations, and nitrites and nitrates indicators were above the norm. The results can be seen in the diagrams in Figures 3, 4, 5, 6 .

Second stage. During this stage of the experiment, the reactor operation time increased and amounted to 16 hours. The experiment lasted 15 days. The composition of the waste liquid, the dose and age of activated sludge, the consumption of waste liquid supplied to the reactor remained the same $-0.2 \mathrm{~m}^{3} /$ day. This time, the reactor operation mode consisted of the following cycles:

1. Filling the system with wastewater;

2. 4 hours 40 minutes - sludge mix aeration, 2 hour 20 minutes - mixing, then 4 hours 40 minutes - aeration and 2 hour 20 minutes - mixing;

3. Sedimentation of the purified water for 2 hours;

4. Drainage of the purified water;

5. Drainage of the excess activated sludge.

At this stage, similarly to the first one, a series of sanitary and chemical analyses were conducted at the end of each cycle. The analyses results showed more effective purification with $91.7 \%$ BOD removal of organic contaminations, while indicators for nitrites, nitrates and ammonia nitrogen are not much lower than those of the previous stage. This indicates that the biological purification processes were more successful than at the previous stage. To achieve the best result, it was decided to increase the plant operation time.

Third stage. During this stage of the experiment, the reactor operation mode was set at 24 hours for 15 days. Again, the waste liquid was composed artificially and was as close to the real waste liquid as possible in its parameters. The wastewater consumption of the plant was $0.20 \mathrm{~m}^{3} /$ day.

The reactor operation mode consisted of the following cycles:

1. Filling the system with wastewater;

2. 7 hours 20 minutes - sludge mix aeration, 3 hour 40 minutes - mixing, then 7 hours 40 minutes - aeration and 3 hour 40 minutes - mixing;

3. Sedimentation of the purified water for 2 hours;

4. Drainage of the purified water;

5. Drainage of the excess activated sludge.

During this stage, the plant showed the best result of the three stages. The BOD removal of organic contaminations is good and amounts to $93 \%$, indicators for nitrites, nitrates and ammonia nitrogen were stable at a low level, which indicates that the biological purification processes were successful. The results of the conducted analyses at all three stages can be seen in the summary diagram presented below (Fig. 3, Fig. 4, Fig. 5, Fig. 6). 


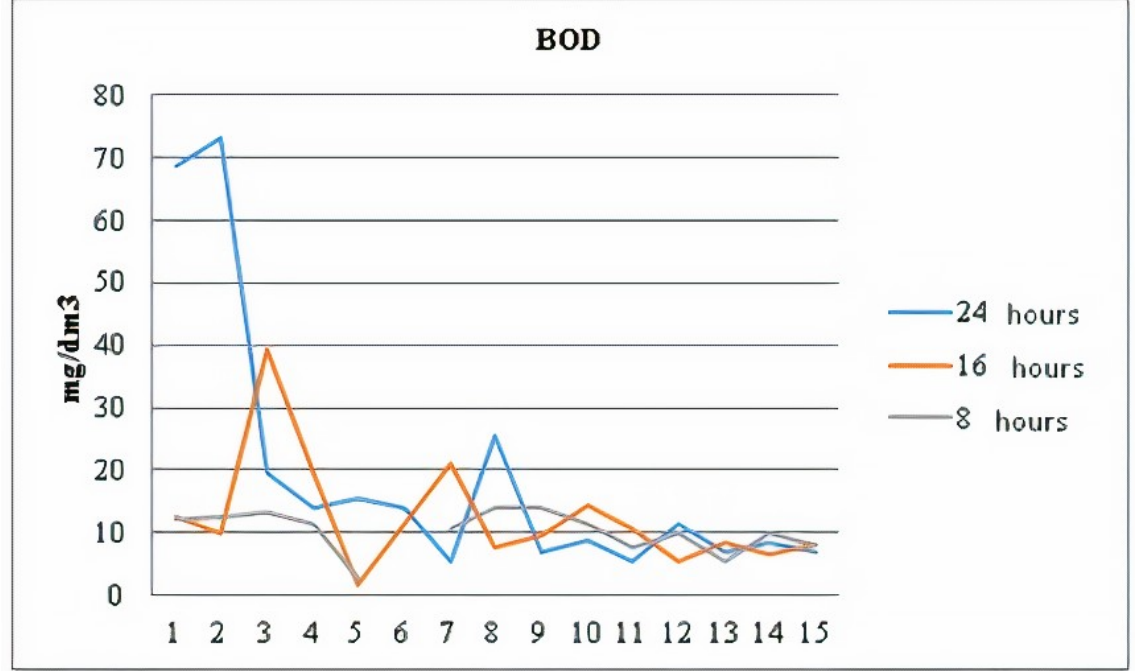

Fig. 3. $\mathrm{BOD}_{5}$ wastewater treatment indicators summary diagram.

This diagram shows that a more or less stable process of wastewater treatment from organic contaminations occurs after 8-9 days of the plant operation in all three time cycles.

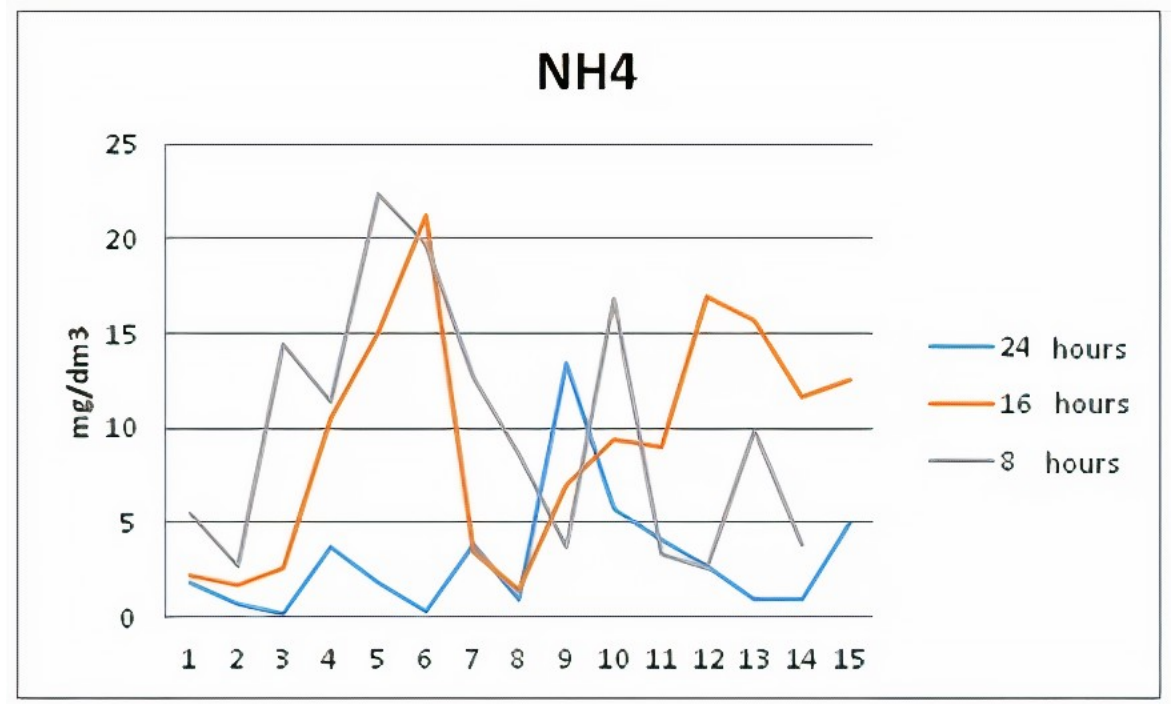

Fig. 4. Ammonia nitrogen-removing wastewater treatment indicators summary diagram.

The diagram shows that the best plant operation for the ammonia nitrogen wastewater treatment is in the 24-hour operation mode. 


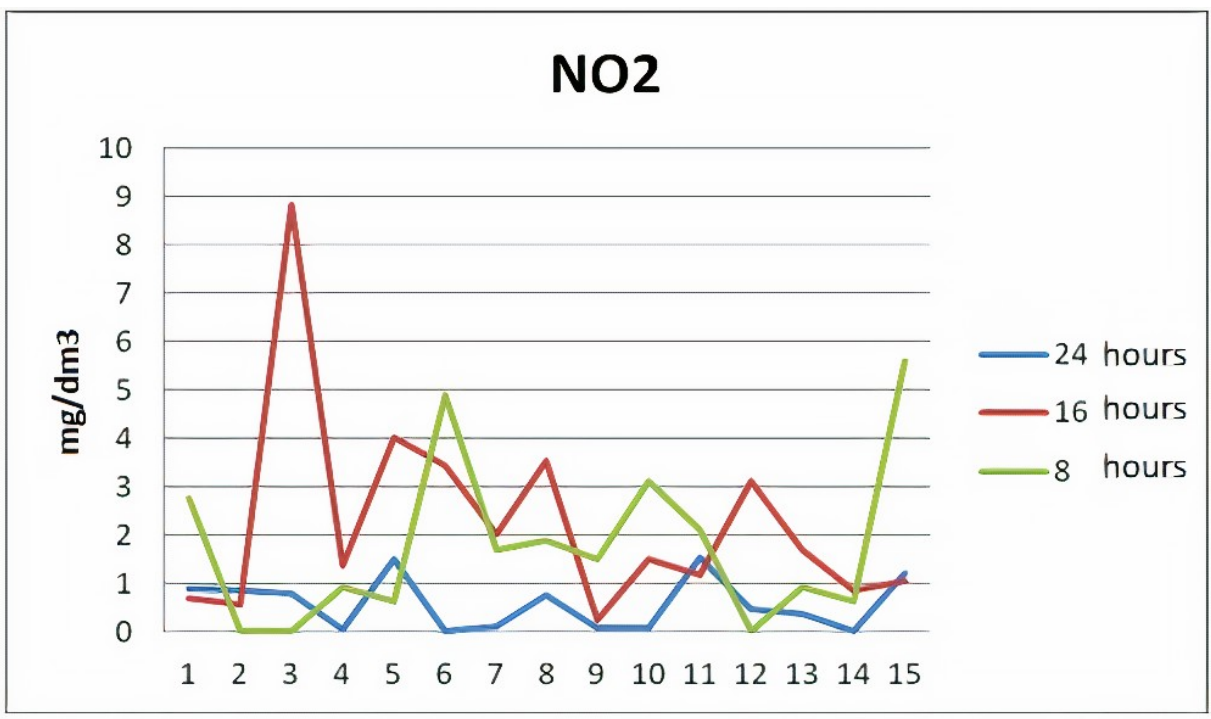

Fig. 5. Nitrites wastewater treatment indicators summary diagram.

According to this diagram it is difficult to talk about any stable plant operation, it only clearly shows that the best result of nitrites wastewater treatment can be obtained in the 24hour plant operation mode.

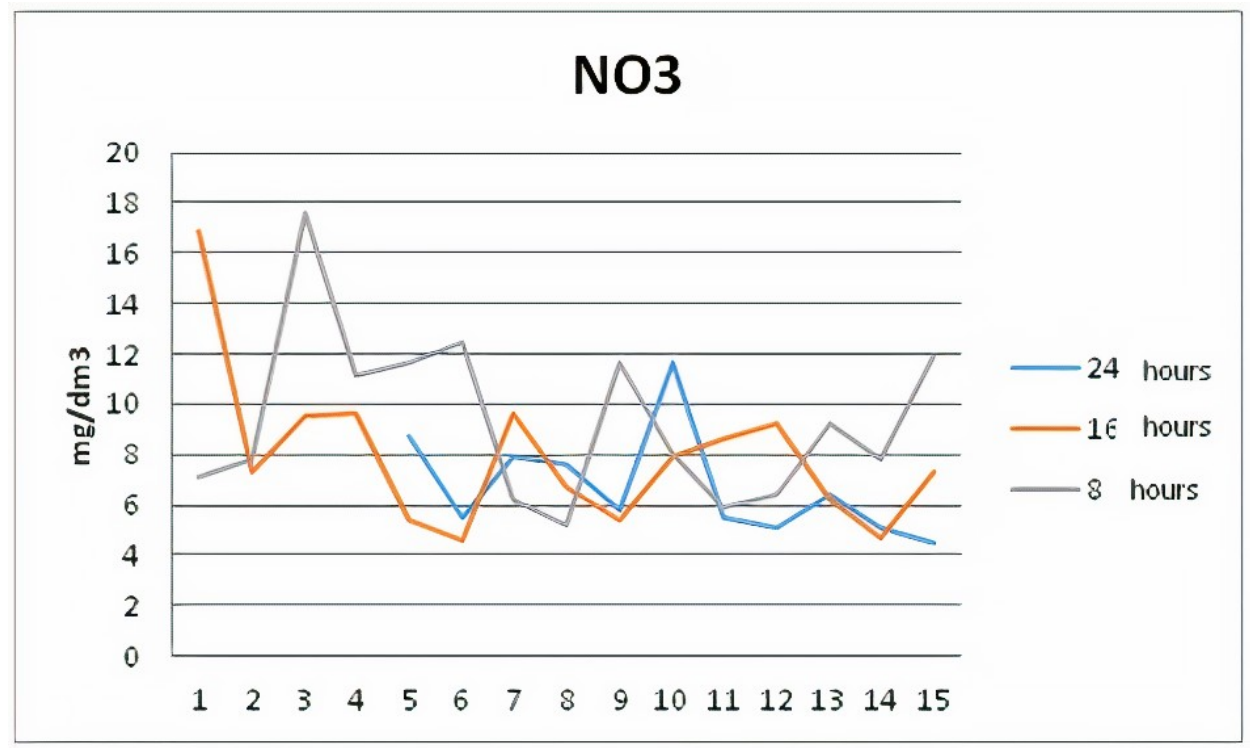

Fig. 6. Nitrates-removing wastewater treatment indicators summary diagram.

This diagram, as well as the ammonia nitrogen-removing wastewater treatment diagram, shows that more or less stable operation of the plant in the 24-hour mode starts after 9-10 days of operation, which is different from other modes. This indicates that nitrifying bacteria appeared in the treated wastewater. They are very sensitive to the slightest changes 
in the oxidized environment, which is associated with the changes in the operation mode of the plant.

The diagrams clearly show how the purification process is progressing and that the most acceptable analysis result is obtained in the 24-hour plant operation mode. The diagram with the results of the $\mathrm{BOD}_{5}$ analyses is smoother, the values are approximately at the same distance, if the average values straight line is drawn.

The main data of the experiment are shown in Table 1.

Table 1. The main data of the experiment.

\begin{tabular}{|l|c|c|c|c|}
\hline \multirow{2}{*}{ Parameters } & Incoming & \multicolumn{3}{|c|}{ Treated drain liquids } \\
\cline { 3 - 5 } & waste liquid & 24 hours & 16 hours & 8 hours \\
\hline $\mathrm{OD}_{5}, \mathrm{mg} / \mathrm{L}$ & 110 & 7.6 & 9.7 & 10 \\
\hline Ammonium nitrogen, $\mathrm{mg} / \mathrm{L}$ & 28 & 1.2 & 7.7 & 9.4 \\
\hline Nitrites, mg/L & - & 0.36 & 2.28 & 1.78 \\
\hline Nitrates, mg/L & - & 5.2 & 7.9 & 9.3 \\
\hline
\end{tabular}

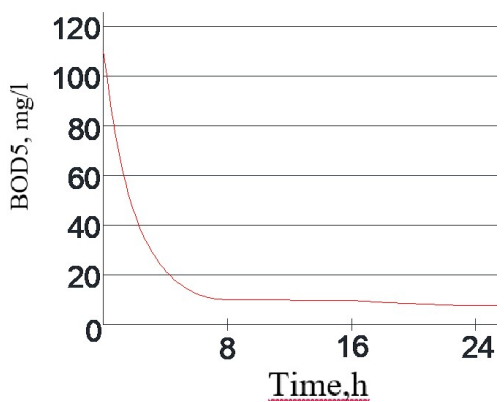

Fig. 7. Diagram of $\mathrm{BOD}_{5}$-removing wastewater treatment dependence from the reactor operation time.

The diagram shows that with the increased time of reactor operation, BOD wastewater treatment is better. The result was obtained at the 24-hour reactor operation time.

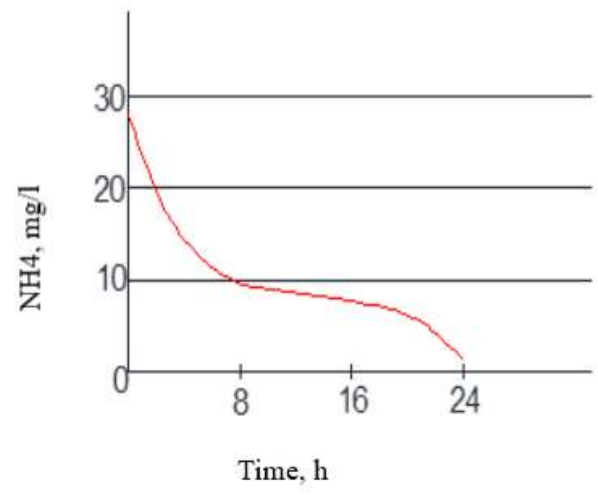

Fig. 8. Diagram of ammonium nitrogen removal dependence from the reactor operation time.

According to this diagram, almost complete removal of ammonium nitrogen occurs in the 24-hour operation mode of the reactor. The diagram shows that during the period of time from 0 to 8 hours, there is a decrease in the concentration of NH4 in water, thus it can be concluded that the nitrification process occurs in the treated water. And in the period of 
time from 8 to 16 hours, the diagram is smoother, which indicates that there are processes of nitrification, but there is no denitrification process yet, and after 16 hours, there is an active process of denitrification, which is displayed in the diagram with sharp decrease in ammonium nitrogen.

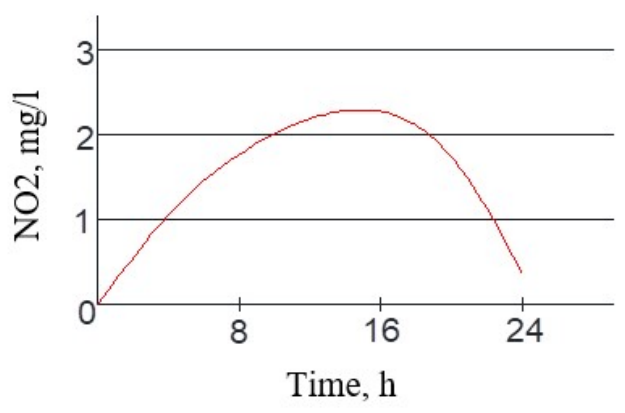

Fig. 9. Diagram of nitrites-removing wastewater treatment dependence from the reactor operation time.

From the diagram of nitrites-removing wastewater treatment dependence from the reactor operation time it can be concluded that maximum concentration of nitrites is reached in the 16-hour operation mode, then concentration of nitrites in treated wastewater decreases.

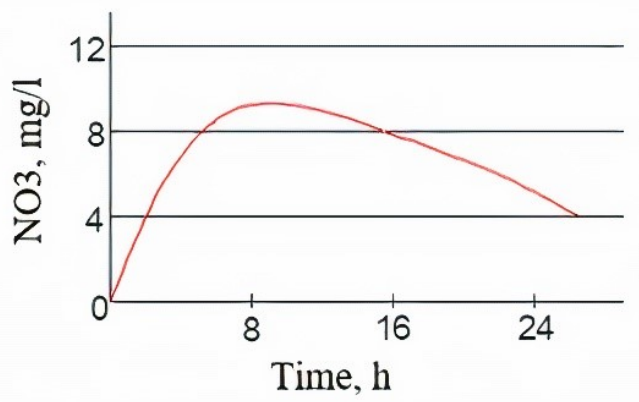

Fig. 10. Diagram of nitrates-removing wastewater treatment dependence from the reactor operation time

If simultaneously analyzing the diagrams of nitrites-removing wastewater treatment dependence from the reactor operation time and the diagram of nitrates-removing wastewater treatment dependence from the reactor operation time one can conclude that active nitrification process occurs when the reactor operation time is up to 8 hours as there are a lot of nitrites and nitrates. Then from 8 to 16 hours nitrites continue to increase and nitrates decrease, this indicates that the nitrification process has reached its peak, and the denitrification process begins. Between 16 and 24 hours, nitrites concentration decreases, nitrates also decrease, which indicates that the nitrification process is over and the denitrification process is active.

\section{Conclusions}

The conducted study has shown that the Sequencing Batch Reactor can potentially be used as a facility for treatment of the wastewater with low concentration of organic contaminations. The laboratory-scale plant operation showed that the 24-hour mode 
treatment allows to purify wastewater not only from organic contamination, but also from nitrogen compounds-ammonium nitrogen, nitrites and nitrates. The received data indicates that deep biological purification processes, which do not cause secondary contamination of the water body when discharging the purified wastewater, occurs in the reactor.

\section{References}

1. Sequencing Batch Reactor. The International Water association publishing. URL: https://www.iwapublishing.com/news/sequencing-batch-reactor

2. Sequencing Batch Reactor Design and Operational Considerations. New England Interstate Water pollution Control commission. September 2005; 1-6.

3. I. M.Panova, I. Noiberg, Ecology Production, 6, 58-61 (2014)

4. T. George, B. Franklin, S. David, Wastewater Engineering Treatment and Reuse (2003)

5. T. H. Quan, N. X. Quyet, E. S. Gogina, Construction - Formation of the environment of vital activity: International interuniversity science.-prakt. conf. students, undergraduates, 1093-1096 (2017)

6. Summary report Sequencing Batch Reactor. United States Environmental Protection Angecy EPA/625/8-86/011. 1986.

7. N. Makisha, D. Semenova, MATEC Web of Conferences, 144, 04016 (2018)

8. O. Ruzhitskaya, E. Gogina, V. Shmalko, (2018), MATEC Web of Conferences, 178, 09015 (2018)

9. Biological nutrient removal (BNR) Operation in Wastewater treatment plants: Manual of Practice No. 29. Water Environment Federation, 2005. Chapter 22.

10. T. H. Quan, E. Gogina, IPB\&KU International symposium on Education and Research in Global Environment Studies in Asia, Bogor, 25 (2018)

11. C. R. Kokare, S. Chakraborty, A. N. Khopade, K. R. Mahadik, Indian J Biotechnol. 8, 159-168 (2009)

12. Nitrification and Denitrification. Michigan Department of Environmental Quality Operator Training and Certification Unit: Online Training Course for Municipal \& Industrial Wastewater.

13. U. V. Voronov, S. V. Yakovlev, Wastewater and Wastewater treatment. (Moscow, MGSU Publishing house ASV, 2006)

14. M. Henze, W. Gujer, T. Mino, M. Loosdrecht, IWA Publishing in its Scientific and Technical Report series (2015)

15. Standard Methods for the Examination of Water and Wastewater. 20th ed. Baltimore, American Public Health Association, 1998.

16. Unified methods for Examination of Water Quality. Part 1 - Methods of chemical analysis of water. Vol. 1 - Basic methods. Moscow, Council for Mutual Economic Assistance Publ., 1987; 1244. (rus.).

17. S. V. Yakovlev, T. A. Karuhina, Biochemical wastewater treatment (Moscow, Stroyizdat Publ., 1980). 